\title{
Nested Convex Bodies are Chaseable
}

\author{
Nikhil Bansal ${ }^{1} \cdot$ Martin Böhm² ${ }^{2}$ Marek Eliáš ${ }^{3}$. Grigorios Koumoutsos ${ }^{4}$. \\ Seeun William Umboh ${ }^{5}$ (i)
}

Received: 21 August 2018 / Accepted: 9 December 2019

(c) Springer Science+Business Media, LLC, part of Springer Nature 2019

\begin{abstract}
In the Convex Body Chasing problem, we are given an initial point $v_{0} \in \mathbb{R}^{d}$ and an online sequence of $n$ convex bodies $F_{1}, \ldots, F_{n}$. When we receive $F_{t}$, we are required to move inside $F_{t}$. Our goal is to minimize the total distance traveled. This fundamental online problem was first studied by Friedman and Linial (DCG 1993). They proved an $\Omega(\sqrt{d})$ lower bound on the competitive ratio, and conjectured that a competitive ratio depending only on $d$ is possible. However, despite much interest in the problem, the conjecture remains wide open. We consider the setting in which the convex bodies are nested: $F_{1} \supset \cdots \supset F_{n}$. The nested setting is closely related to extending the online LP framework of Buchbinder and Naor (ESA 2005) to arbitrary linear constraints. Moreover, this setting retains much of the difficulty of the general setting and captures an essential obstacle in resolving Friedman and Linial's conjecture. In this work, we give a $f(d)$-competitive algorithm for chasing nested convex bodies in $\mathbb{R}^{d}$.
\end{abstract}

Keywords Convex body chasing · Nested convex body chasing · Online algorithms . Competitive analysis

\section{Introduction}

In the convex body chasing problem, introduced by Friedman and Linial [19], we are given an initial position $v_{0} \in \mathbb{R}^{d}$. At each time step $t \geq 1$, we receive a convex set $F_{t} \subset \mathbb{R}^{d}$ as a request, and to serve the request, we must move to some point $v_{t} \in F_{t}$. The goal is to minimize the total distance traveled to serve the requests. The distance can be measured using an arbitrary norm, but unless stated otherwise, it is measured using the Euclidean norm. As any convex body can be approximated arbitrarily well

\footnotetext{
This work was supported by NWO grant 639.022.211, ERC consolidator grant 617951, and GAČR grant P202/12/G061. A preliminary version of this paper appeared in SODA 2018 [7]. N. Bansal, M. Eliáš, G. Koumoutsos and S. W. Umboh: Research was done while the authors were at TU Eindhoven. M. Böhm: Research was done while the author was visiting TU Eindhoven.
}

Extended author information available on the last page of the article 
by intersection of halfspaces, one can assume that $F_{t}$ are halfspaces ${ }^{1}$ and hence this problem is also known as halfspace chasing.

This problem belongs to a very rich class of problems called Metrical Service Systems (MSS) [11]. In an MSS, we are given an arbitrary metric space $(V, \rho)$ and an initial position $v_{0} \in V$. At each time $t$, a request set $F_{t} \subset V$ arrives and we must serve it by moving to some $v_{t} \in F_{t}$. MSS captures several interesting online problems such as the $k$-server problem. While almost tight bounds are known for general MSS $[9,11,18]$, these bounds are not so interesting as typical online problems correspond to MSS with highly structured requests $F_{t}$ and metric space $(V, \rho)$. There has been a lot of interesting work on particular cases of MSS, e.g. [10,16,17,20,23,24], but understanding the role of structure in MSS instances is a major long-term goal in online computation with far-reaching consequences.

Indeed, the main motivation of [19] for considering the convex body chasing problem was to express the competitive ratio of MSS in terms of geometric properties of the request sets $F_{t}$. For the convex body chasing problem, they obtained an $O(1)$ competitive algorithm for $d=2$; for $d>2$, they gave an $\Omega(\sqrt{d})$ lower bound and conjectured that a competitive ratio depending only on $d$ is possible. However, despite much interest, the conjecture remains open.

Nested Convex Body Chasing. In this paper, we consider the nested convex body chasing problem where the requested convex bodies are nested, i.e., $F_{t} \subset F_{t-1}$ for each $t \geq 2$. This natural special case is closely related to many fundamental questions in online algorithms and online learning, and has been of interest in recent years. However, prior to our work, nothing was known for it beyond the results of Friedman and Linial [19] for the general case.

\subsection{Connections and Related Work}

A useful equivalent formulation of the nested problem is the following: Given an initial position $v_{0}$, at each time step $t$, we receive some arbitrary convex body $F_{t}$ (not necessarily nested), and we must move to some point $v_{t}$ that is contained in every convex body seen so far, i.e. $v_{t} \in F_{1} \cap \cdots \cap F_{t}$. The goal is to minimize the total distance traveled. Indeed, this is equivalent to convex body chasing with requests $F_{t}^{\prime}=F_{1} \cap \cdots \cap F_{t}$, which form a nested sequence.

Online Covering LP. The influential primal-dual framework of Buchbinder and Naor for online covering LPs [15] can now be seen as a special case of nested convex body chasing with the $\ell_{1}$-norm. In the former problem, the algorithm starts at the origin $v_{0}=\mathbf{0}$, and at each time $t$, a linear constraint $a_{t}^{\top} x \geq b_{t}$ with non-negative $a_{t}$ and $b_{t}$ arrives. The goal is to maintain a feasible point $x_{t}$ that satisfies all previous requests while the coordinates of $x_{t}$ are only allowed to increase over time. The objective function $c^{\top} x$ (where $c$ is non-negative) can be assumed to be $\|x\|_{1}$ by scaling. Finally, note that in nested convex body chasing with covering constraints, it never helps to

\footnotetext{
${ }^{1}$ If $F$ is the intersection of halfspaces $H_{1}, \ldots, H_{S}$, to simulate the request for $F$, the adversary can give $H_{1}, \ldots, H_{S}$ several times in a round-robin manner until the online algorithm moves inside $F$. Not revealing $F$ directly can only hurt the online algorithm and does not affect the offline solution.
} 
decrease any variable and hence online covering LP is indeed a special case of nested convex body chasing.

While the online primal-dual framework [15] has been applied successfully to many online problems, so far it is limited to LPs with covering and packing constraints, and minor tweaks thereof. An important goal is to extend the online LP framework more broadly beyond packing and covering LPs. For example, it is unclear how to do this even for seemingly simple formulations such as Metrical Task Systems on a line. Since the nested convex body chasing problem corresponds to solving online LPs with arbitrary constraints (with both positive and negative entries) and a specific type of objective, understanding the nested convex body chasing problem is an essential step towards this goal. Indeed, this is one of our main motivations to consider this problem.

General Convex Body Chasing. Another motivation for studying the nested case is that it captures much of the inherent hardness of the general convex body chasing problem. For example, the $\Omega(\sqrt{d})$ lower bound [19] for the general problem also holds in the nested setting. Moreover, several natural algorithms also fail for the nested case.

Other Special Cases. The only known algorithms for chasing convex bodies in $\mathbb{R}^{d}$ with $d>2$ are for certain restricted families of convex bodies $F_{t}$ such as lines and affine subspaces. For chasing lines, Friedman and Linial [19] gave an $O$ (1)-competitive algorithm. For chasing lines and half-lines, Sitters [23] showed that the generalized work function algorithm (WFA) is also $O(1)$-competitive; this is interesting as the WFA is a generic algorithm that attains nearly-optimal competitive ratios for many MSS and is a natural candidate to be $f(d)$-competitive for convex body chasing. Recently, Antoniadis et al. [3] gave an elegant and simple $O(1)$-competitive algorithm for chasing lines, and a $2^{O(d)}$-competitive algorithm for chasing affine subspaces. However, all these results crucially rely on the fact that the requests $F_{t}$ have a lower dimension and do not seem to apply to our problem.

Connections to Online Learning. The convex body chasing problem is also closely related to recent work combining aspects of competitive analysis and online learning. One such work is the Smoothed Online Convex Optimization setting of Andrew et al. $[1,2]$ which incorporates movement cost into the well-studied online learning setting of online convex optimization. The problem is well-understood for $d=1[4,8]$, but nothing is known for larger $d$. Another related work is that of Buchbinder, Chen and Naor [14] which combines online covering LPs with movement cost.

\subsection{Our Results}

Our main result is the following.

Theorem 1 There is an algorithm for chasing nested convex bodies in $\mathbb{R}^{d}$ with competitive ratio that only depends on $d$. In particular, it has competitive ratio $O\left(6^{d}(d !)^{2}\right)$.

The algorithm is described in Sect. 3 and is based on two ideas. First we show that to design an $O_{d}(1)$-competitive algorithm for chasing nested convex bodies, it suffices to make an algorithm for $r$-bounded instances, where all the bodies $F_{t}$ are completely 


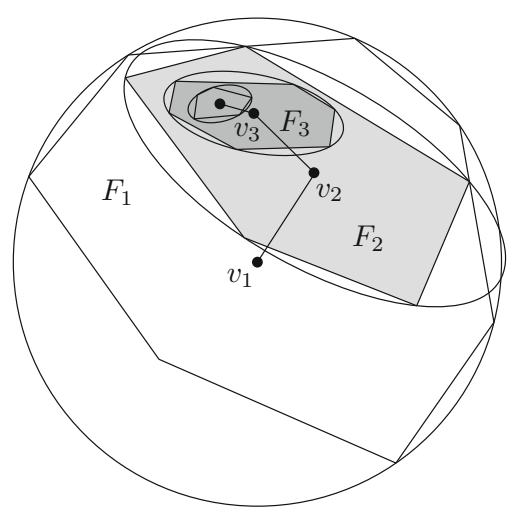

Fig. 1 A few steps of the ellipsoid algorithm

contained in some ball $B(v, r)$ with radius $r$ and center $v$. Moreover, even though competitive ratio is a relative guarantee, it suffices to bound the total movement of the algorithm on any $r$-bounded instance by $O_{d}(r)$. Proving an absolute bound on the distance moved makes the algorithmic task easier and we design such a bounded chasing algorithm in Sect. 3.1.

Surprisingly, the natural approaches for $r$-bounded instances based on the Ellipsoid Method or the centroid approach do not work. In particular, consider a 1-bounded instance where the initial body is $F_{1}=B(\mathbf{0}, 1)$, and the algorithm starts at the origin. As nested convex bodies arrive, if the current point $v_{t-1}$ is infeasible for the request $F_{t}$, a natural approach might be to move to the centroid of $F_{t}$ or to the center of the minimum volume ellipsoid enclosing $F_{t}$ (see Fig. 1). In Sect. 4, we describe a simple 1-bounded instance in $\mathbb{R}^{2}$ on which the above algorithms travel an unbounded distance.

We design our $d$-dimensional bounded chasing algorithm in Sect. 3 based on a recursive approach together with some simple geometric properties. It iteratively invokes the $(d-1)$-dimensional algorithm on at most $d$ bounded instances defined on some suitably chosen hyperplanes. When these instances end, we can argue that the future requests must lie in some smaller ball $B\left(v^{\prime}, \gamma r\right)$, for some fixed $\gamma<1$. Roughly, this allows us to bound the competitive ratio by $g(d)$, that satisfies the recurrence $g(d) \leq d g(d-1) /(1-\gamma)$.

\subsection{Recent Developments}

Since the initial announcement of this work [7], a lot of work was done on this problem with very exciting results. Argue et al. [5] announced an $O(d \log d)$-competitive algorithm for the nested convex body chasing problem. This was further improved by Bubeck et al. [12] who achieved a competitive ratio of $O(\min (d, \sqrt{d \log n}))$, where $n$ is the length of the sequence.

Later, Bubeck et al. [13] obtained an exponential ratio for the general non-nested case. This was subsequently improved to $O(\min (d, \sqrt{d \log n}))$ independently by Argue et al. [6] and Sellke [22]. 


\section{Preliminaries}

We define some notation and recall some basic facts from geometry.

Definition 1 (Nested Convex Body Chasing) In the nested convex body chasing problem in $\mathbb{R}^{d}$, the algorithm starts at some position $v_{0}$, and an online sequence of $n$ nested convex bodies $F_{1} \supset \cdots \supset F_{n}$ arrive one by one. When convex body $F_{t}$ arrives, the algorithm must move to a point $v_{t}$ that lies in $F_{t}$. The goal is to minimize the total distance traveled $\sum_{t=1}^{n}\left\|v_{t}-v_{t-1}\right\|_{2}$.

Note that the choice of measuring distance using the $\ell_{2}$-norm-as opposed to some other symmetric norm, say the $\ell_{1}$-norm-has a negligible effect on the competitive ratio that we obtain because all symmetric norms on $\mathbb{R}^{d}$ are within a $d^{1 / 2}$ factor of each other.

We say that an online deterministic algorithm ALG for some cost-minimization problem $\mathcal{P}$ is $c$-competitive if, for any instance $\mathcal{I} \in \mathcal{P}$, we have

$$
\operatorname{ALG}(\mathcal{I}) \leq c \cdot \operatorname{OPT}(\mathcal{I})+\alpha
$$

where $\operatorname{ALG}(\mathcal{I})$ denotes the cost of the solution to $\mathcal{I}$ produced by the algorithm, $\mathrm{OPT}(\mathcal{I})$ denotes the cost of the optimal solution computed offline (i.e. with prior knowledge of the whole input), and $\alpha$ is some absolute constant independent of the length of the instance $\mathcal{I}$. The constant $c$ is called the competitive ratio of ALG. In the case of nested convex body chasing, the cost corresponds to the distance travelled.

Let $B(v, r)$ denote the ball of radius $r$ centered at $v$. The following useful fact is a variant of John's theorem for balls instead of ellipsoids (for a proof, see e.g. [21, Lemma 8.7.3]):

Proposition 1 (Minimum-volume enclosing ball) Let $F$ be a bounded convex body and suppose $B(v, r)$ is the minimum-volume ball enclosing $F$. Then, the center $v$ of the ball $B(v, r)$ is contained in $F$.

Next we need the following standard fact that we prove here for completeness. We will use it to show that either we can reduce to a $(d-1)$-dimensional instance or a $d$-dimensional instance that is contained in a ball with smaller radius. We use $\mathbf{0}$ to denote the origin.

Proposition 2 (Dimension reduction or radius reduction) Let $d \geq 2$ and $F$ be $a$ bounded convex body in $\mathbb{R}^{d}$ contained in $B(\mathbf{0}, r)$. Then, either $F$ intersects some axis-aligned hyperplane passing through $\mathbf{0}$, or it is contained in some orthant of $B(\mathbf{0}, r)$. Moreover, in the latter case, the smallest ball $B\left(s, r^{\prime}\right)$ enclosing $F$ has radius $r^{\prime} \leq r(1-1 / d)^{1 / 2}$.

Proof The first part immediately follows by convexity. For the second part, we assume, without loss of generality, that $r=1$ and that $F$ is contained in the positive orthant of $B(\mathbf{0}, 1)$. We now show that every point $x$ in the positive orthant of $B(\mathbf{0}, 1)$ is within distance at most $r^{\prime}:=(1-1 / d)^{1 / 2}$ from the point $s=(1 / d, \ldots, 1 / d)$. There are two cases: (1) $\|x\|_{1} \leq 1 ;(2)\|x\|_{1}>1$. 
In case (1), $x$ is in the convex hull of $e_{1}, \ldots, e_{d}$, and $\mathbf{0}$, where $e_{k}$ denotes the $k$-th vector of the standard basis with 1 in the $k$-th coordinate and 0 elsewhere. Therefore, it suffices to prove that $\mathbf{0}$ and $e_{1}, \ldots, e_{d}$ are within distance $r^{\prime}$ from $s$. Indeed, $\|s-\mathbf{0}\|_{2}=$ $(1 / d)^{1 / 2} \leq r^{\prime}($ as $d \geq 2)$ and $\left\|s-e_{k}\right\|_{2}=r^{\prime}$ for each $k \in[d]$.

In case (2), we have

$$
\begin{aligned}
\|s-x\|_{2}^{2} & =\sum_{k=1}^{d}\left(x_{k}-\frac{1}{d}\right)^{2}=\sum_{k=1}^{d}\left(x_{k}^{2}-\frac{2 x_{k}}{d}+\frac{1}{d^{2}}\right) \\
& =\|x\|_{2}^{2}-\frac{2\|x\|_{1}}{d}+\frac{1}{d} \leq 1-1 / d,
\end{aligned}
$$

where the inequality uses that $x \in B(\mathbf{0}, 1)$ and hence $\|x\|_{2} \leq 1$ and $\|x\|_{1}>1$. Thus, the positive orthant of $B(\mathbf{0}, 1)$ is contained in $B\left(s, r(1-1 / d)^{1 / 2}\right)$.

\section{Algorithm}

We now describe our algorithm and prove Theorem 1. We first show, using a guessand-double approach, that finding a good algorithm for the general nested convex body chasing problem can be reduced to an easier problem of designing an algorithm for which we can upper bound the absolute distance traveled, on bounded instances of the following type.

Definition 2 ( $r$-Bounded Instances) An instance with starting point $v_{0}$ and requests $F_{1} \supset \cdots \supset F_{n}$ is said to be $r$-bounded if every request $F_{t}$ is contained in $B\left(v_{0}, r\right)$.

Note that a general instance may not be $r$-bounded for any finite $r$, e.g. in a covering LP where all the $F_{t}$ are halfspaces of the type $a_{t}^{\top} x \geq 1$ where $a_{t}$ has all entries nonnegative.

As we shall see, the task of showing an absolute bound on the distance traveled (instead of a relative bound needed for competitive ratio) makes the problem cleaner. We now describe the reduction.

Lemma 1 (Reduction to Bounded Chasing) Suppose there exists an algorithm Chase $_{d}$ that travels a total distance of at most $g(d) \cdot r$ on $r$-bounded instances for every $r>0$. Then there exists an $f(d)$-competitive algorithm for general instances with $f(d)=8 g(d)$.

Proof Consider a general instance with starting point $v_{0}$. Let $\delta_{t}$ be the distance between $v_{0}$ and the closest point in $F_{t}$; note that $\delta_{t}$ is non-decreasing in $t$ because $F_{t}$ 's are nested. Without loss of generality, we can assume that $v_{0} \notin F_{1}$ and $\delta_{1}=1$ (by scaling).

The algorithm for the general instance proceeds in stages. For $j=1,2, \ldots$, stage $j$ consists of all requests $F_{t}$ for which $\delta_{t} \in\left(2^{j-1}, 2^{j}\right]$, i.e., stage $j$ begins with the first request $F_{t}$ that intersects with $B\left(v_{0}, 2^{j}\right)$ but not with $B\left(v_{0}, 2^{j-1}\right)$, and ends with the last request $F_{t^{\prime}}$ that intersects with $B\left(v_{0}, 2^{j}\right)$.

The algorithm will run a new instance of Chase $d$ at each stage $j$. Let $F_{s(j)}$ be the first request of stage $j$ and $F_{\ell(j)}$ be the last. At the start of stage $j$, the algorithm starts 
at the point $v_{0}$, and begins an instantiation of Chase $e_{d}$ with input sequence

$$
\sigma_{j}=\left(F_{s(j)} \cap B\left(v_{0}, 2^{j}\right), F_{s(j)+1} \cap B\left(v_{0}, 2^{j}\right), \ldots, F_{\ell(j)} \cap B\left(v_{0}, 2^{j}\right)\right) .
$$

After serving the last request $F_{\ell(j)}$, the algorithm returns to $v_{0}$. Note that $\sigma_{j}$ is a $2^{j}$-bounded instance.

We now bound the performance of the algorithm. Clearly, OPT $=\delta_{n}$. Let $j^{*}$ denote the index of the final stage, and hence OPT $\geq 2^{j^{*}-1}$.

For each $1 \leq j \leq j^{*}$, the movement cost during stage $j$ has two parts and can be bounded as follows:

- The movement of Chase $d$ on input $\sigma_{j}$. This is at most $g(d) \cdot 2^{j}$ by the assumed guarantee on Chase . $_{\text {. }}$

- Returning to $v_{0}$ at the end of stage $j$. This cost is at most $2^{j}$ since every request of $\sigma_{j}$ is contained in $B\left(v_{0}, 2^{j}\right)$.

So the total distance traveled by our algorithm is at most

$$
\sum_{j \leq j^{*}} 2^{j}(g(d)+1) \leq 2^{j^{*}+1}(g(d)+1) \leq 4(g(d)+1) \text { OPT } .
$$

The lemma now follows from the fact that $g(d) \geq 1$ as the algorithm might need to travel a distance of $r$.

\subsection{Bounded Chasing Algorithm}

We now focus on designing an algorithm for $r$-bounded instances. The following theorem is our main technical result.

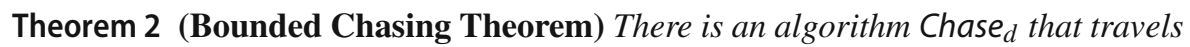
at most $g(d) \cdot r$ distance on $r$-bounded instances where $g(d)=6^{d-1}(d !)^{2}$.

Before we prove Theorem 2, let us note that Theorem 1 immediately follows by combining Theorem 2 and Lemma 1.

We now construct the algorithm Chase $d$ and prove Theorem 2. The proof is by induction on $d$. The base case $(d=1)$ is trivial: the requests form nested intervals and the greedy algorithm that always moves to the closest feasible point is 1-competitive, so $g(d)=1$. In the remainder of this section, we focus on the $d \geq 2$ case and assume that there exists a $(d-1)$-dimensional algorithm Chase $_{d-1}$ with the required properties.

Algorithm. Consider an $r_{0}$-bounded instance with starting point $s_{0}$. The high level idea of the algorithm is to reduce the instance into a sequence of $(d-1)$-dimensional instances and run Chase $_{d-1}$ on these instances.

The algorithm runs in phases. Each phase starts at some center $s$ with radius parameter $r \leq r_{0}$. The first phase starts at $s=s_{0}$ with radius $r=r_{0}$. In each phase, we run Chase $_{d-1}$ with center $s$ and radius $r$ on the $(d-1)$-dimensional instances induced 

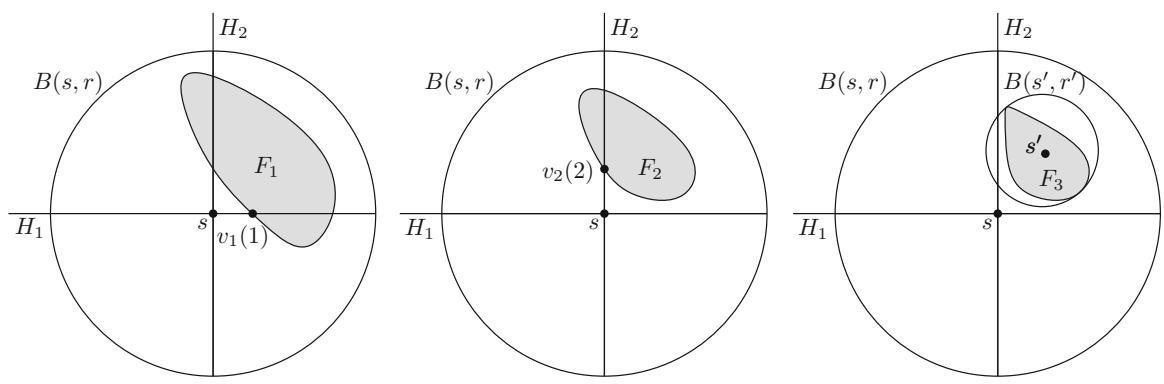

Fig. 2 A phase of Chase 2 , that starts at $s$. The first request $F_{1}$ is served using Chase ${ }_{1}$ in $H_{1}$. The second request $F_{2}$ does not intersect $H_{1}$ so it is served using Chase $e_{1}$ in $H_{2}$. Finally, $F_{3}$ does not intersect $H_{2}$ either and a recentering step is performed

by the $d$ axis-aligned hyperplanes $H_{1}, \ldots, H_{d}$ containing $s$. These are called hyperplane steps. When some request $F_{t}$ arrives that does not intersect with any of these hyperplanes $H_{1}, \ldots, H_{d}$, we perform a recentering step by computing the smallest ball $B\left(s^{\prime}, r^{\prime}\right)$ enclosing $F_{t}$ and moving to $s^{\prime}$; the current phase then ends, and a new phase starts with center $s^{\prime}$ and radius $r^{\prime}$. A key property we will use in the analysis (based on Proposition 2) is that $r^{\prime} \leq r(1-1 / d)^{1 / 2}$, which will allow us to argue that the algorithm makes progress.

Description of a phase. We now describe how a phase works. The reader may find it helpful to refer to Fig. 2 while reading the description below.

Consider a phase that starts at center $s$ and radius $r$. For notational convenience, we reindex the requests so that the first request of the phase is $F_{1}$. Let $H_{1}, \ldots, H_{d}$ denote the axis-aligned hyperplanes passing through $s$.

Hyperplane steps. Initially at request $F_{1}$, we choose the axis-aligned hyperplane $H_{k}$ with the smallest index $k \in[d]$ that intersects $F_{1}$ (if no such hyperplane exists, we move to the Recentering step below), and run Chase ${ }_{d-1}$ on the $(d-1)$-dimensional instance induced by $H_{k}$ and follow it for as long as we can. More specifically, we run Chase $_{d-1}$ on the $(d-1)$-dimensional instance with starting point $s$ and radius $r$, and requests

$$
F_{1} \cap H_{k}, \ldots, F_{\ell(k)} \cap H_{k},
$$

where $F_{\ell(k)}$ is the last request in the current phase that intersects $H_{k}$; for $t \leq \ell(k)$, we serve request $F_{t}$ by moving to $v_{k}(t)$ where $v_{k}(t)$ is the location of Chase $e_{-1}$ on request $F_{t} \cap H_{k}$.

When the first request $F_{t}$ arrives that does not intersect the current $H_{k}$, i.e., $F_{t}=$ $F_{\ell(k)+1}$, then we change the hyperplane and repeat the above process. That is, we pick $H_{k^{\prime}}$ that intersects $F_{t}$, with the smallest index $k^{\prime}$ (if it exists), and run Chase ${ }_{d-1}$ on $H_{k^{\prime}}$ starting at position $s$ with radius $r$ and requests $F_{\ell(k)+1} \cap H_{k^{\prime}}, \ldots$ and follow it for as long as we can.

Recentering step. If a request $F_{t}$ arrives that does not intersect any $H_{k}$ for $k \in[d]$, we compute the smallest ball $B\left(s^{\prime}, r^{\prime}\right)$ containing $F_{t}$, move to $s^{\prime}$ and serve $F_{t}$ (note 
that $s^{\prime} \in F_{t}$ by Proposition 1). The current phase ends, and a new phase with center $s^{\prime}$ and radius $r^{\prime}$ starts.

This completes the description of a phase and we now turn to analyzing the algorithm.

Analysis. We need to show that Chase $_{d}$ is feasible (Claim 1) and bound the distance it travels (Claim 2). These claims give us Theorem 2.

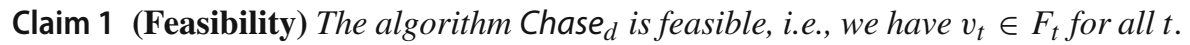

Proof We prove the claim by induction on $d$. For $d=1$, the algorithm is trivial and it is always feasible. Assume that the claim is true for $d-1$. Consider some request $F_{t}$. Observe that Chase ${ }_{d}$ either performs a hyperplane step or a recentering step. In the former, since we follow Chase ${ }_{d-1}$ on some hyperplane $H_{k}$ and Chase ${ }_{d-1}$ stays feasible (by induction), we have that $v_{t} \in F_{t} \cap H_{k}$. In the latter, $v_{t}$ is the center of the smallest ball containing $F_{t}$ so $v_{t} \in F_{t}$ by Proposition 1 . Thus, in both cases, $v_{t} \in F_{t}$.

Next, we bound the distance traveled by Chase $_{d}$.

Claim 2 (Cost) The total distance traveled by Chase on $_{\text {an }} r$-bounded instance is at most $g(d) \cdot r$, where $g(d)=6^{d-1}(d !)^{2}$.

Proof We now bound the distance traveled during each phase. Consider phase $j$. Let $B_{j}$ denote Chase ${ }_{d}$ 's enclosing ball during the phase and $r_{j}$ be its radius. Note that during the phase, the algorithm stays within $B_{j}$ and all requests $F_{t}$ in the phase are contained in $B_{j}$. The movement in phase $j$ consists of:

- Movement due to hyperplane steps. On each hyperplane $H_{k}$, we move at most $g(d-1) \cdot r_{j}$ by following Chase $_{d-1}$. Thus, the total movement due to hyperplane steps is at most $d \cdot g(d-1) \cdot r_{j}$.

- Movement due to switching hyperplanes. We switch hyperplanes at most $d-1$ times, so this is at most $(d-1) \cdot 2 r_{j}$.

- Movement due to recentering. This is at most $2 r_{j}$.

Thus, the total distance traveled in phase $j$ is at most

$$
d \cdot(g(d-1)+2) \cdot r_{j} \leq 3 d \cdot g(d-1) \cdot r_{j},
$$

since $g(d-1) \geq 1$.

We now bound the sum of $r_{j}$ over all phases. By Proposition 1 , the radii of the enclosing balls decrease geometrically across phases: $r_{j} \leq r_{j-1} \cdot \sqrt{1-1 / d}$ for $j>1$. Thus, the sum of $r_{j}$ over all phases is at most $r_{1} \cdot \sum_{j=1}^{\infty}(\sqrt{1-1 / d})^{j}=r_{1} \cdot \frac{1}{1-\sqrt{1-1 / d}}$. As $r_{1}=r$, the total distance traveled by Chase $_{d}$ over all phases is at most

$$
\begin{aligned}
3 d \cdot g(d-1) \cdot \frac{r}{1-(1-1 / d)^{1 / 2}} & \leq 3 d \cdot g(d-1) \cdot 2 d r \\
& =6 d^{2} g(d-1) r
\end{aligned}
$$


where we use that $1-(1-1 / d)^{1 / 2} \geq 1 /(2 d)$, as $(1-x)^{\alpha} \leq 1-\alpha x$ for any $x \in[0,1]$ and $\alpha \in[0,1]$.

Thus, we conclude that Chase $_{d}$ travels at most $g(d) \cdot r$, where $g(d)=6^{d-1}(d !)^{2}$ is the solution to the recurrence relation $g(d)=6 d^{2} \cdot g(d-1)$ with base case $g(1)=1$.

\section{Lower Bounds for Ellipsoid and Centroid}

In this section, we consider some natural ellipsoid-based and centroid-based algorithms for chasing nested bodies in the $r$-bounded setting, and show that they are not competitive, i.e., their competitive ratio can be arbitrarily large if the length of the input sequence approaches inifinty. The main reason these algorithms fail is that for (relatively) flat convex bodies, the center of the bounding ellipsoid, or the centroid, can move by a large distance in directions that do not matter.

Henceforth, for a set $S \subset \mathbb{R}^{d}$, let $E(S)$ denote the smallest-volume ellipsoid containing $S$. Consider the following ellipsoid-based algorithm Ellipsoid: for each request $F_{t}$, if the current position is not in $F_{t}$, move to the center of $E\left(F_{t}\right)$.

We now construct an $\mathbb{R}^{2}$ instance in which Ellipsoid travels an arbitrarily large distance while the optimal offline cost is at most 1 . In the following, we will use the notation $(x, y)$ for a point in $\mathbb{R}^{2}$.

The starting point of the instance is $(0,1)$. Each request $F_{t}$ is an intersection of four halfspaces $A, B, C, H_{t}$. The first three halfspaces $A, B, C$ are $y \geq 0, x \geq-1$, and $x \leq 1$, respectively. The last halfspace $H_{t}$ will be different for each $F_{t}$.

For the first request $F_{1}$, we set

$$
H_{1}=\{(x, y) \mid 2 y \leq(1-\alpha) x+(1+\alpha)\},
$$

for some parameter $\alpha$. Note that the boundary of $H_{1}$ passes through the points $(-1, \alpha)$ and $(1,1)$, as seen in Fig. 3. The parameter $\alpha$ is chosen so that the center of $E\left(F_{1}\right)$ is strictly to the right of the $y$ axis, as guaranteed by the following lemma:

Lemma 2 There exists $0<\alpha<1$ such that the center of the smallest ellipsoid containing $F_{1}$ has a strictly positive $x$-coordinate. More precisely, its center is $(c, b)$ with $c, b>0$.

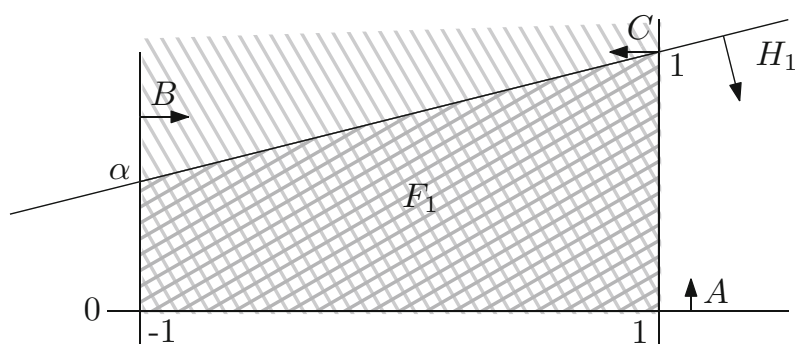

Fig. 3 The first request $F_{1}$ 


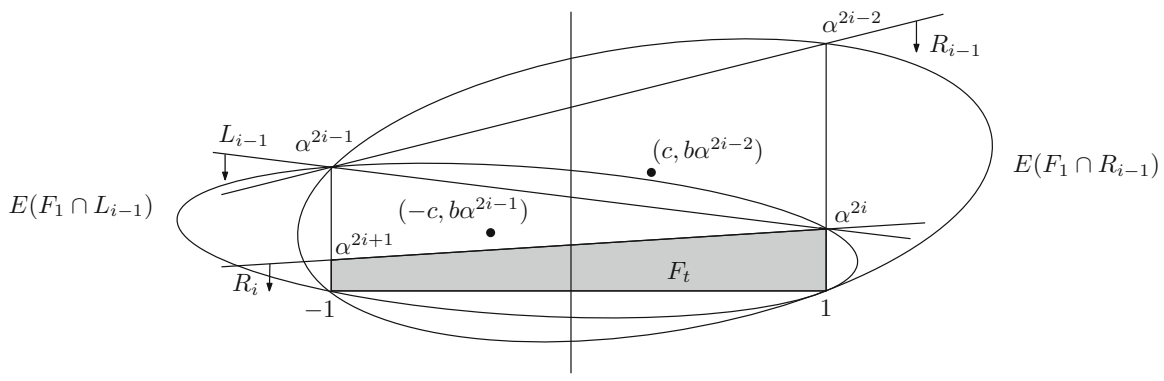

Fig. 4 Constructing the sequence of $R_{i}$ and $L_{i}$ for $i=1,2, \ldots$

We postpone the proof of Lemma 2 and continue with the description of the request sequence. The remaining nested bodies $\left\{F_{t} \mid t \geq 2\right\}$ are created so that the $x$-coordinate of the center of $E\left(F_{t}\right)$ oscillates between $c$ and $-c$. To this end, we construct two infinite families of halfspaces $R_{i}$ and $L_{i}$ : for $i \geq 0$, we define

$$
\begin{aligned}
& R_{i}:=\left\{(x, y) \mid 2 y \leq\left(\alpha^{2 i}-\alpha^{2 i+1}\right) x+\left(\alpha^{2 i}+\alpha^{2 i+1}\right)\right\} \\
& L_{i}:=\left\{(x, y) \mid 2 y \leq\left(\alpha^{2 i+2}-\alpha^{2 i+1}\right) x+\left(\alpha^{2 i+2}+\alpha^{2 i+1}\right)\right\} .
\end{aligned}
$$

Now observe that the boundary of $R_{i}$ passes through the points $\left(1, \alpha^{2 i}\right)$ and $\left(-1, \alpha^{2 i+1}\right)$, and the boundary of $L_{i}$ passes through $\left(-1, \alpha^{2 i+1}\right)$ and $\left(1, \alpha^{2 i+2}\right)$. See Fig. 4 for an illustration.

We now describe the requests $F_{t}$ for $t \geq 2$. For even $t$, we set $H_{t}$ to be $L_{i}$, where $i$ is the smallest index such that $L_{i}$ does not contain the current position of the algorithm. For odd $t$, we select $H_{t}$ to be $R_{i}$ in a similar fashion. This completes our description of the requests $F_{t}$. Looking at Fig. 4 , one can easily observe that our requests $F_{t}$ are indeed nested.

The following lemma describes the position of the center of each ellipsoid $E\left(F_{t}\right)$. Note that $c$ and $b$ are the strictly positive constants from Lemma 2.

Lemma 3 When $t$ is odd, the center of $E\left(F_{t}\right)$ is $\left(c, b \alpha^{2 i}\right)$ for some $i$. When $t$ is even, the center of $E\left(F_{t}\right)$ is $\left(-c, b \alpha^{2 i+1}\right)$ for some $i$.

Proof Recall that $F_{t}=A \cap B \cap C \cap H_{t}$. First, consider odd $t$, where $H_{t}=R_{i}$. We define a map $f:(x, y) \rightarrow\left(x, y / \alpha^{2 i}\right)$, which rescales the $y$-coordinate. Note that it maps $F_{t}$ to $F_{1}$. Moreover, $f$ preserves ratios between volumes, and therefore the map of the smallest ellipsoid containing $F_{t}$ is the smallest ellipsoid containing $F_{1}$. We know that its center is at $(c, b)$, and therefore the center of $E\left(F_{t}\right)$ is at $\left(c, b \alpha^{2 i}\right)$.

For $t$ even, we have $H_{t}=L_{i}$ for some $i$. We define $g:(x, y) \rightarrow\left(-x, y / \alpha^{2 i+1}\right)$, which first mirrors $F_{t}$ with respect to the $y$ axis, and then rescales the $y$-coordinate, so that $g\left(F_{t}\right)=F_{1}$. Clearly, mirroring preserves the volumes, while rescaling preserves their ratios. Therefore, $f$ maps $E\left(F_{t}\right)$ to $E\left(F_{1}\right)$ whose center is at $(c, b)$ and the center of $E\left(F_{t}\right)$ is at $\left(-c, b \alpha^{2 i+1}\right)$.

Let us now estimate the competitive ratio of Ellipsoid. At each time step, it incurs cost at least $2 c$, since it moves between two points with $x$-coordinates $c$ and $-c$ respec- 
tively. Therefore, if $N$ is the total number of requests, the total cost incurred by Ellipsoid is at least $N \cdot 2 c$, which can be arbitrarily large. On the other hand, the point $(0,0)$ is contained in every $F_{t}$, since it belongs to $F_{1}$ and also to every halfspace $R_{i}$ and $L_{i}$. Therefore, the cost of OPT is at most 1 and the competitive ratio of Ellipsoid is unbounded.

Proof of Lemma 2 Using a computer algebra system, we computed that for $\alpha=1 / 2$ the center of $E\left(F_{1}\right)$ is at $(0.24568,0.40571)$. This can be calculated, e.g., using the function ellipsoidhull in R, but similar functions are also available for MATLAB. This shows that $\alpha=1 / 2$ satisfies the requirements of the lemma.

The manual computation of $E\left(F_{1}\right)$ for $\alpha=1 / 2$ is laborious, but we can still give a formal proof of the existence of a suitable $\alpha$. Let $F_{1}(\alpha)$ denote $F_{1}$ with parameter $\alpha$. Observe that $F_{1}(0)$ is a triangle with vertices $(1,0),(1,1)$, and $(-1,0)$; and $F_{1}(1)$ is a rectangle with vertices $(1,0),(1,1),(-1,0)$ and $(-1,1)$. Since the center of $E\left(F_{1}(\alpha)\right)$ evolves continuously with $\alpha$, it suffices to show that the center of the smallest ellipsoid containing the triangle $F_{1}(0)$ lies strictly to the right of the $y$-axis. By continuity, this implies that there exists $\alpha>0$ such that the $x$-coordinate of the center of $E\left(F_{1}(\alpha)\right)$ is still strictly positive.

We define the affine map $f:(x, y) \rightarrow\left(\begin{array}{l}1-1 \\ 0 \sqrt{3}\end{array}\right)(x, y)^{\top}$. Let $T=F_{1}(0)$. This transformation makes $T$ equilateral by first shearing it to the left to be symmetric with respect to the $y$-axis and then shrinking the $y$-coordinate. The smallest ellipsoid containing an equilateral triangle is its circumcircle, whose center lies in the intersection of its altitudes. Since one of the altitudes lies on the $y$-axis, the $x$-coordinate of the center of $E(f(T))$ is 0 , and its $y$-coordinate is strictly positive. Since $f$ preserves ratios between volumes, we have $f(E(T))=E(f(T))$. Therefore, applying $f^{-1}$ to the center of $E(f(T))$, we know that the center of $E(T)$ has both coordinates strictly positive.

Lower bound for the centroid algorithm. Similar to the ellipsoid-based algorithm, one can consider the following centroid-based algorithm: for each request $F_{t}$, if the current position is not in $F_{t}$, move to the centroid (center of mass) of $F_{t}$.

The same sequence of requests $F_{t}$ as above also shows that this algorithm is not competitive either. In fact, the analysis here is much easier, as we can compute the centroids using simple geometry (the input convex bodies can be partitioned into a right triangle and a rectangle, as seen e.g. in Fig. 3).

A simple calculation shows that for $\alpha=1 / 2$, the centroid of $F_{1}$ is $(1 / 9,7 / 9)$. For the convex bodies requested later, the $x$-coordinate of the centroid will oscillate between $-1 / 9$ and 1/9, again showing that the total distance traveled by the algorithm can be made arbitrarily large.

Acknowledgements We would like to thank Sébastien Bubeck, Niv Buchbinder, Anupam Gupta, Guru Guruganesh, Cristóbal Guzmán, and René Sitters for several interesting discussions.

\section{References}

1. Andrew, L.H., Barman, S., Ligett, K., Lin, M., Meyerson, A., Roytman, A., Wierman, A.: A tale of two metrics: simultaneous bounds on competitiveness and regret. In: Proceedings of COLT 2013, pp. $741-763$ (2013) 
2. Andrew, L.H., Barman, S., Ligett, K., Lin, M., Meyerson, A., Roytman, A., Wierman, A.: A tale of two metrics: simultaneous bounds on competitiveness and regret. ArXiv e-prints, arXiv:1508.03769 (2015)

3. Antoniadis, A., Barcelo, N., Nugent, M., Pruhs, K., Schewior, K., Scquizzato, M.: Chasing convex bodies and functions.In: Proceedings of LATIN 2016, pp. 68-81 (2016)

4. Antoniadis, A., Schewior, K.: A tight lower bound for online convex optimization with switching costs. In: Proceedings of WAOA 2017, pp. 164-175 (2017)

5. Argue, C.J., Bubeck, S., Cohen, M.B., Gupta, A., Lee, Y.T.: A nearly-linear bound for chasing nested convex bodies. In: Proceedings of SODA 2019, pp. 117-122 (2019)

6. Argue, C.J., Gupta, A., Guruganesh, G., Tang, Z.: Chasing convex bodies with linear competitive ratio. ArXiv e-prints, arXiv:1905.11877 (2019)

7. Bansal, N., Böhm, M., Eliáš, M., Koumoutsos, G., Umboh, S.W.: Nested convex bodies are chaseable. In: Proceedings of SODA 2018, pp. 1253-1260 (2018)

8. Bansal, N., Gupta, A., Krishnaswamy, R., Pruhs, K., Schewior, K., Stein, C.: A 2-competitive algorithm for online convex optimization with switching costs. In: Proceedings of APPROX/RANDOM 2015, pp. 96-109 (2015)

9. Bartal, Yair, Bollobás, Béla, Mendel, Manor: Ramsey-type theorems for metric spaces with applications to online problems. J. Comput. Syst. Sci. 72(5), 890-921 (2006)

10. Bartal, Yair, Koutsoupias, Elias: On the competitive ratio of the work function algorithm for the k-server problem. Theor. Comput. Sci. 324(2), 337-345 (2004)

11. Borodin, Allan, Linial, Nathan, Saks, Michael E.: An optimal on-line algorithm for metrical task system. J. ACM 39(4), 745-763 (1992)

12. Bubeck, S., Lee, Y. T., Li, Y., Sellke, M.: Chasing nested convex bodies nearly optimally. ArXiv e-prints, arXiv:1811.00999 (2018)

13. Bubeck, S., Lee, Y.T., Li, Y., Sellke, M.: Competitively chasing convex bodies. In: Proceedings of STOC 2019, pp. 861-868 (2019)

14. Buchbinder, N., Chen, S., Naor, J.: Competitive analysis via regularization. In: Proceedings of SODA 2014, pp. 436-444 (2014)

15. Buchbinder, Niv, Naor, Joseph: Online primal-dual algorithms for covering and packing. Math. Oper. Res. 34(2), 270-286 (2009)

16. Burley, William R.: Traversing layered graphs using the work function algorithm. J. Algorithms 20(3), 479-511 (1996)

17. Chrobak, M., Larmore, L.L.: Metrical task systems, the server problem and the work function algorithm. In: Fiat, A., Gerhard, J. (eds.) Online Algorithms: The State of the Art, pp. 74-96. Springer, Berlin (1998)

18. Fiat, A., Mendel, M.: Better algorithms for unfair metrical task systems and applications. In: Proceedings of STOC 2000, pp. 725-734 (2000)

19. Friedman, Joel, Linial, Nathan: On convex body chasing. Discrete Comput. Geom. 9, 293-321 (1993)

20. Koutsoupias, Elias, Papadimitriou, Christos H.: On the k-server conjecture. J. ACM 42(5), 971-983 (1995)

21. Matoušek, Jiří, Gärtner, Bernd: Understanding and Using Linear Programming. Springer, Berlin (2007)

22. Sellke, M.: Chasing convex bodies optimally. ArXiv e-prints, arXiv:1905.11968 (2019)

23. Sitters, R.: The generalized work function algorithm is competitive for the generalized 2-server problem. SIAM J. Comput. 43(1), 96-125 (2014)

24. Sitters, R.A., Stougie, L.: The generalized two-server problem. J. ACM 53(3), 437-458 (2006)

Publisher's Note Springer Nature remains neutral with regard to jurisdictional claims in published maps and institutional affiliations.

\section{Affiliations}

\section{Nikhil Bansal ${ }^{1} \cdot$ Martin Böhm $^{2} \cdot$ Marek Eliáśs $^{3} \cdot$ Grigorios Koumoutsos $^{4}$. Seeun William Umboh ${ }^{5}$}


$\bowtie \quad$ Seeun William Umboh

william.umboh@sydney.edu.au

Nikhil Bansal

bansal@gmail.com

Martin Böhm

martin.boehm@uni-bremen.de

Marek Eliáš

marek.elias@epfl.ch

Grigorios Koumoutsos

gkoumout@ulb.ac.be

1 Centrum Wiskunde and Informatica, CWI, Amsterdam, Netherlands

2 University of Bremen, Bremen, Germany

3 École polytechnique fédérale de Lausanne, Lausanne, Switzerland

4 Université Libre de Bruxelles, Brussels, Belgium

5 The University of Sydney, Sydney, Australia 